\title{
Phase estimation with photon number constraint
}

\author{
Masahito HAYASHI \\ Graduate School of Information Sciences, Tohoku University \\ Centre for Quantum Technologies, National University of Singapore
}

\begin{abstract}
Many researches proposed the use of the noon state as the input state for phase estimation, which is one topic of quantum metrology. This is because the input noon state provides the maximum Fisher information at the specific point. However, the Fisher information does not necessarily give the attainable bound for estimation error. In this paper, we adopt the local asymptotic mini-max criterion as well as the mini-max criterion, and show that the maximum Fisher information does not give the attainable bound for estimation error under these criteria in the phase estimation. We also propose the optimal input state under the constraints for photon number of the input state instead of the noon state.
\end{abstract}

\section{KEYWORDS}

Phase estimation, photon number constraint, maximum photon number, asymptotic limit, quantum metrology, average of square of photon number

\section{Introduction}

As one of the most simple methods in quantum metrology, phase estimation has been treated by many authors [1]-[6] and its experimental demonstration has been reported by many groups [7]-[10]. As is pointed by [11], the phase estimation with Mach-Zehnder (MZ) interferometer is important from the view point of the standard configuration. Many experiment groups realized the noon state. The reason is why the noon state provides the optimal Fisher information. In the independent and identical distributed setting of state estimation, Fisher information provides the asymptotic bound of mean square error (MSE) [12]-[14]. However, the phase estimation does not fall in this setting. Hence, it has not been discussed sufficiently whether the minimum estimation error is realized when the noon state is inputed.

In this paper, we treat the phase estimation with two kinds of photon number constraints. One is the constraint for the average of the square of the photon number concerning the input state. The other is the constraint for the maximum photon number concerning the

Received October 28, 2010; Revised January 3, 2011; Accepted January 12, 2011.

hayashi@math.is.tohoku.ac.jp

DOI: $10.2201 / \mathrm{NiiPi} .2011 .8 .9$ input state. In order to treat the phase estimation, we focus on two criteria. One is the minimum mean square error (MSE) under the locally unbiased (LUB) condition, which coincides with the SLD Fisher information [15]-[17]. The other is the mini-max value of the MSE, which minimizes the worst MSE. The latter is essentially equivalent with the optimization with the covariant restriction for measurement [18]. As an intermediate concept, we focus on the local asymptotic mini-max criterion, which was introduced by Hajek [19] and was applied to quantum channel estimation by Hayashi [20]. Then, we can define three kinds of bounds under two kinds of constraint. The main purpose of this paper is the comparison between these three bounds with both constraints. The second purpose is to check the validity of use of the noon state as the input state and seeking the optimal input state.

The organization of this paper is the following. In section 2 , we treat the phase estimation problem under the locally unbiased condition. In section 3, we clarify the relation between the single-application case and the multiple-application case. In section 4, we discuss the locally asymptotic mini-max criterion. In section 5 , we treat the global mini-max criterion and the relation among three kinds of criteria under the asymptotic 
limit. In section 6, we treat the MSE of the noon state with both of the global and local asymptotic mini-max criteria.

\section{SLD Fisher information and locally unbiased condition}

In the two-mode photonic system $L^{2}\left(\mathbb{R}^{2}\right)$, the phase operator $U_{\theta}$ is given by

$$
U_{\theta}:=\sum_{n, m \geq 0} e^{i(n-m) \theta}|n, m\rangle\langle n, m|
$$

with $\theta \in[-\pi, \pi]$. When the true parameter $\theta$ is unknown, we can estimate the parameter by inputing the known state $|\phi\rangle$ and measuring the output state $U_{\theta}|\phi\rangle$. Hence, when the measurement corresponds to the POVM $M=\left\{M_{\hat{\theta}}\right\}$, our estimator is described by the pair of the input state $|\phi\rangle$ and the POVM $M$ as Fig. 1.

In this case, the estimate $\hat{\theta}$ obeys the distribution $\left\langle\phi\left|U_{\theta}^{\dagger} M_{\hat{\theta}} U_{\theta}\right| \phi\right\rangle$. The mean square error (MSE) is given by

$$
E_{\theta}(|\phi\rangle, M):=\int_{-\pi}^{\pi}\left(\theta-T_{\theta}(\hat{\theta})\right)^{2}\left\langle\phi\left|U_{\theta}^{\dagger} M_{\hat{\theta}} U_{\theta}\right| \phi\right\rangle d \hat{\theta},
$$

where

$$
T_{\theta}(\hat{\theta}):= \begin{cases}\hat{\theta}+2 \pi & \text { if } \hat{\theta}<\theta-\pi \\ \hat{\theta} & \text { if } \theta-\pi \leq \hat{\theta}<\theta+\pi \\ \hat{\theta}-2 \pi & \text { if } \theta+\pi \leq \hat{\theta} .\end{cases}
$$

When the input state $U_{\theta}|\phi\rangle$ is given, our problem is estimation of the parameter $\theta$ with the pure state family $\left\{U_{\theta}|\phi\rangle\langle\phi| U_{\theta}^{\dagger}\right\}$. In order to treat the lower bound of the MSE, we focus on the symmetric logarithmic derivative (SLD) Fisher information [21]

$$
J_{\theta}(|\phi\rangle)=4\left\langle\phi\left|\frac{d U_{\theta}^{\dagger}}{d \theta} \frac{d U_{\theta}}{d \theta}\right| \phi\right\rangle-4\left|\left\langle\phi\left|\frac{d U_{\theta}}{d \theta}\right| \phi\right\rangle\right|^{2} .
$$

This equation can be shown by the following way: $\frac{d}{d \theta} U_{\theta}|\phi\rangle\left\langle\phi\left|U_{\theta}^{\dagger}=X\right| \phi\right\rangle\langle\phi|+| \phi\rangle\langle\phi| X^{\dagger}$, where $X=\frac{d U_{\theta}}{d \theta}-$ $\left\langle\phi\left|\frac{d U_{\theta}}{d \theta}\right| \phi\right\rangle$. Thus, $\frac{d}{d \theta} U_{\theta}|\phi\rangle\langle\phi| U_{\theta}^{\dagger}=2\left(X|\phi\rangle\langle\phi|+| \phi\rangle\langle\phi| X^{\dagger}\right) \circ$ $|\phi\rangle\langle\phi|$, where $A \circ B=\frac{A B+B A}{2}$. Then, $J_{\theta}(|\phi\rangle)=$ $\operatorname{Tr}\left(2\left(X|\phi\rangle\langle\phi|+| \phi\rangle\langle\phi| X^{\dagger}\right)\right)^{2}|\phi\rangle\langle\phi|$, which equals the right hand side of (1).

In statistical inference, an unbiased estimator plays an important role. However, since the parameter space

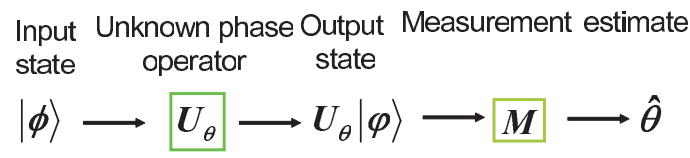

Fig. 1 Scheme of phase estimation. is the interval $[-\pi, \pi]$, it is difficult to define the unbiased condition. It is possible to define the locally unbiased condition. When the estimator $(|\phi\rangle, M)$ satisfies the condition

$$
\begin{aligned}
& \int_{-\pi}^{\pi} T_{\theta}(\hat{\theta})\left\langle\phi\left|U_{\theta_{0}}^{\dagger} M_{\hat{\theta}} U_{\theta_{0}}\right| \phi\right\rangle d \hat{\theta}=\theta_{0} \\
& \left.\int_{-\pi}^{\pi} T_{\theta}(\hat{\theta}) \frac{d}{d \theta}\left\langle\phi\left|U_{\theta}^{\dagger} M_{\hat{\theta}} U_{\theta}\right| \phi\right\rangle\right|_{\theta=\theta_{0}} d \hat{\theta}=1,
\end{aligned}
$$

it is called locally unbiased at $\theta_{0}$. When an estimator $(|\phi\rangle, M)$ is locally unbiased (LUB) at $\theta_{0}$, the SLD Cramer-Rao inequality

$$
E_{\theta}(|\phi\rangle, M) \geq J_{\theta}(|\phi\rangle)^{-1} .
$$

Its proof is similar to the usual case of the SLD CramerRao inequality [15]-[17] because the Schwarz inequality plays the same rule.

In this problem, when we input the state with a larger photon number, the MSE becomes smaller. So, it is suitable to constraint the photon number of the input state. As the first kind of constraint, we restrict the average of the square photon number $\left(N^{2}\right)_{a v}(|\phi\rangle):=$ $\left\langle\phi\left|\hat{N}^{2}\right| \phi\right\rangle$ of the input state $|\phi\rangle$, where $\hat{N}:=\sum_{n, m}(n+$ $m)|n, m\rangle\langle n, m|$. As the second kind of constraint, we restrict the maximum of the photon number $N_{\max }(|\phi\rangle):=$ $\max _{n, m}\{n+m \mid\langle n, m \mid \phi\rangle \neq 0\}$ of the input state $|\phi\rangle$. So, we define the two bounds of MSE as follows:

$$
\begin{aligned}
& \tilde{C}_{a v, \theta}(E) \\
& :=\inf \left\{\begin{array}{l|l}
E_{\theta}(|\phi\rangle, M) & \begin{array}{l}
(|\phi\rangle, M) \text { is LUB at } \theta_{0} \\
\left(N^{2}\right)_{a v}(|\phi\rangle) \leq E^{2}
\end{array}
\end{array}\right\} \\
& \tilde{C}_{\max , \theta}(E) \\
& :=\inf \left\{\begin{array}{l|l}
E_{\theta}(|\phi\rangle, M) & \begin{array}{l}
(|\phi\rangle, M) \text { is LUB at } \theta_{0} \\
N_{\max }(|\phi\rangle) \leq E
\end{array}
\end{array}\right\} .
\end{aligned}
$$

Since $J_{\theta}(|\phi\rangle) \leq 4\left\langle\phi\left|\hat{N}^{2}\right| \phi\right\rangle$, the SLD Cramer-Rao inequality (2) implies

$$
\tilde{C}_{\max , \theta}(E) \geq \tilde{C}_{a v, \theta}(E) \geq \frac{1}{4 E^{2}} .
$$

When $E$ is an integer $n$ and the input state $|\phi\rangle$ is the noon state $\left|\phi_{n, \text { noon }}\right\rangle:=\frac{1}{\sqrt{2}}(|n, 0\rangle+|0, n\rangle), J_{\theta}\left(\left|\phi_{n, \text { noon }}\right\rangle\right)^{-1}=\frac{1}{4 n^{2}}$. There exists a POVM satisfying the locally unbiased condition whose MSE attains the inverse of the SLD Fisher information. Hence,

$$
\tilde{C}_{\max , \theta}(n)=\tilde{C}_{a v, \theta}(n)=\frac{1}{4 n^{2}} .
$$

In the state estimation, it is known that there exists a sequence of estimators that asymptotically attains the inverse of the SLD Fisher information at all points [12]-[14]. Hence, we expect that the bounds

$$
\tilde{C}_{\max , \theta}:=\lim _{E \rightarrow \infty} E^{2} \tilde{C}_{\max , \theta}(E)=\frac{1}{4}
$$




$$
\tilde{C}_{a v, \theta}:=\lim _{E \rightarrow \infty} E^{2} \tilde{C}_{a v, \theta}(E)=\frac{1}{4}
$$

can be attained asymptotically in all points by a single sequence of estimator under the respective restrictions. However, the same argument does not necessarily hold in the case of channel estimation.

\section{Multiple application}

As an extension of the formulation given in Section 2 , we assume that the $m$-fold tensor product system of $L^{2}\left(\mathbb{R}^{2}\right)$, i.e., $L^{2}\left(\mathbb{R}^{2}\right)^{\otimes m}$, is allowed as the input system and the unknown operator $U_{\theta}^{\otimes m}$ is applied. It is required to estimate the unknown parameter $\theta$. In this case, we consider the number operator $\hat{N}_{m}:=\sum_{k=1}^{m} I^{\otimes k-1} \otimes \hat{N} \otimes$ $I^{\otimes m-k}$, and the estimator is given by the pair of the input state $|\phi\rangle$ on $L^{2}\left(\mathbb{R}^{2}\right)^{\otimes m}$ and a POVM $M$ on $L^{2}\left(\mathbb{R}^{2}\right)^{\otimes m}$. Now, we define the projection

$$
\begin{aligned}
P_{a, b}:= & \sum_{\# 1} \sum_{\# 2}\left|a_{1}, b_{1}\right\rangle\left\langle a_{1}, b_{1}\right| \otimes \cdots \\
& \otimes\left|a_{m}, b_{m}\right\rangle\left\langle a_{m}, b_{m}\right|,
\end{aligned}
$$

where \#1 is $a_{1}, \ldots, a_{m}: \sum_{j} a_{j}=a$ and \#2 is $b_{1}, \ldots, b_{m}$ : $\sum_{j} b_{j}=b$. Then, we define the normalized vector $\left|\phi_{a, b}\right\rangle:=\frac{1}{\| P_{a, b}|\phi\rangle \|} P_{a, b}|\phi\rangle$ and the isometry $V_{m}:=$ $\sum_{a, b}|a, b\rangle\left\langle\phi_{a, b}\right|$ from $L^{2}\left(\mathbb{R}^{2}\right)^{\otimes m}$ to $L^{2}\left(\mathbb{R}^{2}\right)$. Then, the pair of the state $V_{m}|\phi\rangle$ and the POVM $\left\{V_{m} M_{\hat{\theta}} V_{m}^{\dagger}\right\}$ gives an estimator on the single input system $L^{2}\left(\mathbb{R}^{2}\right)$. The outcome of this estimator has the same statistical behavior of the original estimator $(|\phi\rangle, M)$ on $L^{2}\left(\mathbb{R}^{2}\right)^{\otimes m}$. Further, the average of the square number of $V_{m}|\phi\rangle$ equals that of $|\phi\rangle$. Conversely, any estimator $\left(|\phi\rangle,\left\{M_{\hat{\theta}}\right\}\right)$ on the single input system $L^{2}\left(\mathbb{R}^{2}\right)$ can be simulated by an estimator $\left(W_{m}^{\dagger}|\phi\rangle,\left\{W_{m}^{\dagger} M_{\hat{\theta}} V_{m}\right\}\right)$ on $L^{2}\left(\mathbb{R}^{2}\right)^{\otimes m}$, where $W_{m}:=\sum_{a, b}\left|\psi_{a, b}\right\rangle\langle a, b|$ and $\left|\psi_{a, b}\right\rangle$ is a normalized vector on the range of $P_{a, b}$. So, the optimization of estimator $\left(|\phi\rangle,\left\{M_{\hat{\theta}}\right\}\right)$ on the single input system $L^{2}\left(\mathbb{R}^{2}\right)$ with constraint concerning average of square of photon number is mathematically equivalent with that on the $m$-fold tensor product system $L^{2}\left(\mathbb{R}^{2}\right)^{\otimes m}$ with constraint concerning average of square of photon number.

Next, we consider the case when only state in the subspace spanned by $\{|00\rangle,|10\rangle,|01\rangle\}$ is allowed as the input system. In this case, the input system is 3dimensional and is written by $\mathcal{H}$. Its $m$-fold input system is given as $\mathcal{H}^{\otimes m}$. For a given input state $|\phi\rangle \in$ $\mathcal{H}^{\otimes m}$, the state $V_{m}|\phi\rangle$ satisfies the condition $\max _{a, b}\{a+$ $\left.b \mid\left\langle a, b\left|V_{m}\right| \phi\right\rangle \neq 0\right\} \leq m$. Conversely, when the input state $|\phi\rangle$ satisfies the condition $\max _{a, b}\{a+b \mid\langle a, b \mid \phi\rangle \neq$ $0\} \leq m$, and when $\left|\psi_{a, b}\right\rangle$ is chosen from the intersection the range of $P_{a, b}$ and $\mathcal{H}^{\otimes m}$, the state $W_{m}|\phi\rangle$ belongs to $\mathcal{H}^{\otimes m}$. The estimator $\left(W_{m} \mid \phi,\left\{W_{m} M_{\hat{\theta}} W_{m}^{\dagger}\right\}\right)$ simulates the estimator $\left(|\phi\rangle,\left\{M_{\hat{\theta}}\right\}\right)$. So, the optimization of estimator on the single input system $L^{2}\left(\mathbb{R}^{2}\right)$ with constraint concerning maximum of photon number is mathematically equivalent with that on the $m$-fold tensor product system $\mathcal{H}$.

Overall, multiple application setting is a physical situation different from single application setting. This is because multiple application requires multiple application on the same system $L^{2}\left(\mathbb{R}^{2}\right)$. However, this setting can be mathematically simulated by single application setting. This kind of simulation plays an important role in the next section.

\section{Locally asymptotic mini-max crite- rion}

In this section, we consider the locally asymptotic mini-max criterion. In statistics, we often treat minimax criterion, in which, we optimize the worst case. In the following, we optimize the worst MSE among the $\epsilon$ neighborhood of the given point $\theta_{0}$. We treat the asymptotic behavior of this optimum value and consider the limit $\epsilon \rightarrow 0$ concerning the asymptotic coefficient. That is, we define

$$
\begin{aligned}
& C_{a v, \theta}:=\lim _{\epsilon \rightarrow 0} \lim _{E \rightarrow \infty} E^{2} C_{a v, \theta}(E, \epsilon) \\
& C_{a v, \theta}(E, \epsilon):=\inf _{|\phi\rangle}\left\{C_{\theta}(|\phi\rangle: \epsilon) \mid\left(N^{2}\right)_{a v}(|\phi\rangle) \leq E^{2}\right\} \\
& C_{\theta_{0}}(|\phi\rangle: \epsilon):=\inf _{M} \sup _{\theta \in U\left(\epsilon, \theta_{0}\right)} E_{\theta}(|\phi\rangle, M) \\
& C_{\max , \theta}:=\lim _{\epsilon \rightarrow 0} \lim _{E \rightarrow \infty} E^{2} C_{\max , \theta}(E, \epsilon) \\
& C_{\max , \theta}(E, \epsilon):=\inf _{|\phi\rangle}\left\{C_{\theta}(|\phi\rangle: \epsilon) \mid N_{\max }(|\phi\rangle) \leq E\right\} .
\end{aligned}
$$

We also define similar values with multiple application.

$$
\begin{aligned}
& C_{a v, \theta_{0}, m}(E, \epsilon) \\
& \quad:=\inf _{(|\phi\rangle, M)}\left\{\sup _{\theta \in U\left(\epsilon, \theta_{0}\right)} E_{\theta}(|\phi\rangle, M) \mid\left(N_{m}^{2}\right)_{a v}(|\phi\rangle) \leq E^{2} m^{2}\right\} \\
& C_{\text {max }, \theta_{0}, m} \\
& \left.\quad:=\inf _{(|\phi\rangle, M)}\left\{\sup _{\theta \in U\left(\epsilon, \theta_{0}\right)} E_{\theta}(|\phi\rangle, M)|| \phi\right\rangle \in \mathcal{H}^{\otimes m}\right\},
\end{aligned}
$$

where $(|\phi\rangle, M)$ is an estimator on $L^{2}\left(\mathbb{R}^{2}\right)^{\otimes m}$. As is discussed in Section 3, estimators on $L^{2}\left(\mathbb{R}^{2}\right)^{\otimes m}$ can be simulated by estimators on $L^{2}\left(\mathbb{R}^{2}\right)$ with large photon number states. Hence,

$$
\begin{aligned}
& C_{a v, \theta}=\lim _{\epsilon \rightarrow 0} \lim _{m \rightarrow \infty} m^{2} E^{2} C_{a v, \theta, m}(1, \epsilon) \\
& C_{\text {max }, \theta}=\lim _{\epsilon \rightarrow 0} \lim _{m \rightarrow \infty} m^{2} C_{\max , \theta, m} .
\end{aligned}
$$

Further, Proposition 1 of [20] implies that

$$
\begin{aligned}
& \lim _{\epsilon \rightarrow 0} \lim _{m \rightarrow \infty} m^{2} C_{a v, \theta, m}(1, \epsilon) \leq \tilde{C}_{a v, \theta} \\
& \lim _{\epsilon \rightarrow 0} \lim _{m \rightarrow \infty} m^{2} C_{\max , \theta, m} \leq \tilde{C}_{\max , \theta} .
\end{aligned}
$$


That is,

$$
\begin{aligned}
& C_{a v, \theta} \leq \tilde{C}_{a v, \theta} \\
& C_{\text {max }, \theta} \leq \tilde{C}_{\text {max }, \theta} .
\end{aligned}
$$

\section{Global mini-max criterion}

Next, we consider global optimization, i.e., global mini-max criterion. For this purpose, we consider group covariant condition for our POVM $M$. In this optimization with photon number constraint, we can restrict the input state on the subspace $\mathcal{H}_{1}$ spanned by $\{|n, 0\rangle\} \cup\{|0, n\rangle\}$. A POVM $M=\left\{M_{\hat{\theta}}\right\}$ is covariant when there exists a vector $|T\rangle$ such that $M_{\hat{\theta}} d \hat{\theta}=\frac{1}{2 \pi} U_{\hat{\theta}}|T\rangle\langle T| U_{\hat{\theta}}^{\dagger} d \hat{\theta}$, where $|T\rangle=\sum_{n=0}^{\infty} e^{i \theta_{n}}|n, 0\rangle+$ $\sum_{n=1}^{\infty} e^{i \theta_{n}^{\prime}}|0, n\rangle$, and $\theta_{n}$ and $\theta_{n}$ are arbitrary coefficients. As is shown by Holevo [18], since the stochastic behavior of the error $\hat{\theta}-\theta$ of the covariant POVM does not depend on the true parameter $\theta$, any input state $|\phi\rangle$ satisfies

$$
\inf _{M} \max _{\theta} E_{\theta}(|\phi\rangle, M)=\inf _{M: \operatorname{cov}} \max _{\theta} E_{\theta}(|\phi\rangle, M) .
$$

Hence, when we optimize the worst value $\max _{\theta} E_{\theta}(|\phi\rangle, M)$, it is sufficient to treat the covariant POVM. That is, our estimator is written by the pair of input state $|\phi\rangle=\sum_{n=0}^{\infty} a_{n}|n, 0\rangle+\sum_{n=1}^{\infty} a_{-n}|0, n\rangle$ and $|T\rangle=\sum_{n=0}^{\infty} e^{i \theta_{n}}|n, 0\rangle+\sum_{n=1}^{\infty} e^{i \theta_{n}^{\prime}}|0, n\rangle$, where $\vec{a}$ $=\left\{a_{n}\right\}_{n=-\infty}^{\infty} \in L^{2}(\mathbb{Z})$. This estimator is simulated by the pair of the input state $\left|\phi^{\prime}\right\rangle=\sum_{n=0}^{\infty} e^{-i \theta_{n}} a_{n}|n, 0\rangle+$ $\sum_{n=1}^{\infty} e^{-i \theta_{n}^{\prime}} a_{-n}|0, n\rangle$ and the covariant POVM given by $\left|T_{0}\right\rangle=\sum_{n=0}^{\infty}|n, 0\rangle+\sum_{n=1}^{\infty}|0, n\rangle$. This is because $\left|\left\langle T\left|U_{\hat{\theta}}^{\dagger} U_{\theta}\right| \phi\right\rangle\right|^{2}=\left|\left\langle T_{0}\left|U_{\hat{\theta}}^{\dagger} U_{\theta}\right| \phi^{\prime}\right\rangle\right|^{2}$. Hence, it is sufficient to treat the case with $|T\rangle=\left|T_{0}\right\rangle$. In this case, we define $a_{n}^{\prime}:=e^{-i \theta_{n}} a_{n}$ for $n \geq 0$ and $a_{-n}^{\prime}:=e^{-i \theta_{n}^{\prime}} a_{-n}$ for $n>0$. Then, when the error $\hat{\theta}-\theta$ obeys the square $\left|\mathcal{F}^{-1}\left(\vec{a}^{\prime}\right)(\theta)\right|^{2}$ of the inverse Fourier transform $\mathcal{F}^{-1}\left(\vec{a}^{\prime}\right)(\theta):=\sum_{n=-\infty}^{\infty} a_{n}^{\prime} e^{-i n \theta}$ of discrete series of $\vec{a}^{\prime}=\left\{a_{n}^{\prime}\right\}_{n=-\infty}^{\infty}$.

Thus, we obtain

$$
\begin{aligned}
C(|\phi\rangle) & :=\inf _{M} \sup _{\theta} E_{\theta}(|\phi\rangle, M) \\
& =\inf _{\overrightarrow{a^{\prime}}:\left|a_{n}^{\prime}\right|=\left|a_{n}\right|}\left\{\frac{1}{2 \pi} \int_{-\pi}^{\pi} \hat{\theta}^{2}\left|\mathcal{F}^{-1}\left(\overrightarrow{a^{\prime}}\right)(\hat{\theta})\right|^{2} d \hat{\theta}\right\}
\end{aligned}
$$

and

$$
\begin{gathered}
C_{a v}(E) \\
:=\inf _{(\mid \phi, M)}\left\{\sup _{\theta} E_{\theta}(|\phi\rangle, M) \mid\left\langle\phi\left|\hat{N}^{2}\right| \phi\right\rangle \leq E^{2}\right\} \\
=\inf _{\vec{a}:\|\vec{a}\|=1}\left\{\frac{1}{2 \pi} \int_{-\pi}^{\pi} \hat{\theta}^{2}\left|\mathcal{F}^{-1}(\vec{a})(\hat{\theta})\right|^{2}\right. \\
\left.\times d \hat{\theta} \mid\left(N^{2}\right)_{a v}(\vec{a}) \leq E^{2}\right\}
\end{gathered}
$$

$$
\begin{gathered}
C_{\max }(E) \\
:=\inf _{(\mid \phi, M)}\left\{\sup _{\theta} E_{\theta}(|\phi\rangle, M) \mid\left\langle\phi\left|\hat{N}^{2}\right| \phi\right\rangle \leq E^{2}\right\} \\
=\inf _{\vec{a}:\|\vec{a}\|=1}\left\{\frac{1}{2 \pi} \int_{-\pi}^{\pi} \hat{\theta}^{2}\left|\mathcal{F}^{-1}(\vec{a})(\hat{\theta})\right|^{2}\right. \\
\left.\times d \hat{\theta} \mid N_{\max }(\vec{a}) \leq E\right\},
\end{gathered}
$$

where $\left(N^{2}\right)_{a v}(\vec{a}) \quad:=\quad \sum_{n} n^{2}\left|a_{n}\right|^{2}, \quad N_{\max }(\vec{a}) \quad:=$ $\max _{n}\left\{|n| \mid a_{n} \neq 0\right\}$, and $\|\vec{a}\|^{2}:=\sum_{n=-\infty}^{\infty}\left|a_{n}\right|^{2}$.

From the definitions, we obtain

$$
\begin{aligned}
& C(|\phi\rangle) \geq C_{\theta}(|\phi\rangle: \epsilon) \\
& C_{a v}(E) \geq C_{a v, \theta}(E, \epsilon) \\
& C_{\max }(E) \geq C_{\max , \theta}(E, \epsilon),
\end{aligned}
$$

and the asymptotic limits are defined by

$$
\begin{aligned}
& C_{a v}:=\lim _{E \rightarrow \infty} E^{2} C_{a v}(E) \\
& C_{\text {max }}:=\lim _{E \rightarrow \infty} E^{2} C_{\text {max }}(E) .
\end{aligned}
$$

Given an arbitrary sequence $a_{E}=\left\{a_{E, n}\right\}_{n=-\infty}^{\infty}$ of elements of $L^{2}(\mathbb{Z})$, we focus on the function

$$
f(x):=\lim _{E \rightarrow \infty} \sqrt{E} a_{E, E x} .
$$

Then, the relations (5) and (6) imply that

$$
\begin{aligned}
& C_{a v}=\inf _{f \in L^{2}(\mathbb{R}):\|f\|=1}\left\{\left\langle f\left|P^{2}\right| f\right\rangle \mid\left\langle f\left|Q^{2}\right| f\right\rangle \leq 1\right\} \\
& C_{\text {max }}=\inf _{f \in L^{2}([-1,1]):\|f\|=1}\left\langle f\left|P^{2}\right| f\right\rangle,
\end{aligned}
$$

where $Q$ and $P$ are the position and momentum operators on $L^{2}(\mathbb{R})$. The equation (12) is the same as the result obtained by Imai and Hayashi [3].

Next, we focus on the specific sequence of the input states $\left|\phi_{E}\right\rangle$ whose coefficient satisfies (10). Similar to (11) and (12), we obtain

$$
\lim _{E \rightarrow \infty} E^{n} C\left(\left|\phi_{E}\right\rangle\right)=\inf _{g \in L^{2}(\mathbb{R}):|g(x)|=|f(x)|}\left\langle g\left|P^{2}\right| g\right\rangle .
$$

The uncertainly relation implies that

$$
C_{a v}=\frac{1}{4}
$$

and the infimum is attained when $f(x)=e^{-\frac{x^{2}}{4}}$. The latter is given by

$$
C_{\text {max }}=\frac{\pi^{2}}{4}
$$

and the infimum is attained when $f(x)=$ $(2 \pi)^{1 / 4} \sin \frac{\pi(1+x)}{2}$. Comparing the right hand sides 
of (5) and (6) with those of (11) and (12), the bounds $C_{a v}$ and $C_{\max }$ can be attained by sequence of input states

$$
c_{1}\left(\sum_{n=0}^{\infty} e^{-\frac{n^{2}}{4 E^{2}}}|n, 0\rangle+\sum_{n=1}^{\infty} e^{-\frac{n^{2}}{4 E^{2}}}|0, n\rangle\right)
$$

and

$$
\begin{aligned}
& c_{2}\left(\sum_{n=0}^{E} \sin \frac{\pi(E+n+1)}{2 E+2}|n, 0\rangle\right. \\
& \left.\quad+\sum_{n=1}^{E} \sin \frac{\pi(E+n+1)}{2 E+2}|0, n\rangle\right),
\end{aligned}
$$

where $c_{1}$ and $c_{2}$ are normalized constants.

From the relations (8) and (9), we obtain

$$
\begin{aligned}
& C_{a v} \geq C_{a v, \theta} \\
& C_{\text {max }} \geq C_{\text {max }, \theta} .
\end{aligned}
$$

As is shown in Proposition 3 of Hayashi [20], the bounds $C_{a v, \theta}=\lim _{\epsilon \rightarrow 0} \lim _{m \rightarrow \infty} m^{2} E^{2} C_{a v, \theta, m}(\epsilon, E)$ and $C_{\max , \theta}=\lim _{\epsilon \rightarrow 0} \lim _{m \rightarrow \infty} m^{2} C_{\max , \theta, m}$ can be globally attained by two-step methods [12] [13] asymptotically. Since any estimator on the multiple system $L^{2}\left(\mathbb{R}^{2}\right)^{\otimes m}$ can be simulated by a suitable estimator on the single input system $L^{2}\left(\mathbb{R}^{2}\right)$, the opposite inequalities of (16) and (17) hold. That is, combining (3) and (4), we obtain

$$
\begin{aligned}
& C_{a v, \theta}=C_{a v}=\frac{1}{4}=\tilde{C}_{a v, \theta} \\
& C_{\max , \theta}=C_{\max }=\frac{\pi^{2}}{4}>\frac{1}{4}=\tilde{C}_{\max , \theta} .
\end{aligned}
$$

So, when we adopt the maximum photon number constraint, there is a non-negligible difference between the local asymptotic mini-max bound and the bound with locally unbiased condition.

\section{Analysis on noon state}

Now, we consider the asymptotic limit of the performance with the locally asymptotic min-max criterion when the noon state $\frac{1}{\sqrt{2}}(|n, 0\rangle+|0, n\rangle)$ is inputed and the covariant measurement is applied. In this case, first, we fix an arbitrary small real number $\epsilon>0$ and focus on the neighborhood $U\left(\epsilon, \theta_{0}\right)$ for an arbitrary point $\theta_{0}$. When $n>2 \pi / \epsilon$, the relation $U_{\theta} \frac{1}{\sqrt{2}}(|n, 0\rangle+|0, n\rangle)=$ $U_{\theta+2 \pi / n} \frac{1}{\sqrt{2}}(|n, 0\rangle+|0, n\rangle)$ holds and $\theta$ and $\theta+\frac{2 \pi}{n}$ belongs to $U\left(\epsilon, \theta_{0}\right)$. That is, we cannot distinguish two parameters $\theta$ and $\theta+\frac{2 \pi}{n}$. Even if we could estimate the unknown parameter $\theta \bmod \frac{2 \pi}{n}$ perfectly, it is not easy to estimate the parameter $\theta$ in the parameter space $U\left(\epsilon, \theta_{0}\right)$. In the following, we consider the case of $\theta=\theta_{0}-\epsilon \bmod \frac{2 \pi}{n}$. Assume that we could the unknown parameter $\theta \bmod$ $\frac{2 \pi}{n}$. In this case, there are still $K:=\left\lfloor\frac{n \epsilon}{\pi}\right\rfloor$ candidates with the width $\frac{2 \pi}{n}$ in the parameter space $U\left(\epsilon, \theta_{0}\right)$. Assume that, we decide the true parameter to be $\frac{2 \pi}{n} i+\theta_{0}-\epsilon$ with the probability $p_{i}$. Then, the mini-max error is evaluated as follows.

$$
\begin{aligned}
& \max _{j=1, \ldots, K} \sum_{i=1}^{K} p_{i}\left(\frac{1}{2 n \pi}\right)^{2}(j-i)^{2} \\
&=\left(\frac{2 \pi}{n}\right)^{2} \max _{j=1, \ldots, K}\left(j^{2}-2 j \sum_{i=1}^{K} i p_{i}+\sum_{i=1}^{K} i^{2} p_{i}\right) \\
&=\left(\frac{2 \pi}{n}\right)^{2}\left(\max _{j=1, \ldots, K}\left(j-\sum_{i=1}^{K} i p_{i}\right)^{2}\right. \\
&\left.+\sum_{i=1}^{K} i^{2} p_{i}-\left(\sum_{i=1}^{K} i p_{i}\right)^{2}\right) \\
& \geq\left(\frac{2 \pi}{n}\right)^{2} \max _{j=1, \ldots, K}\left(j-\sum_{i=1}^{K} i p_{i}\right)^{2} \\
& \geq\left(\frac{2 \pi}{n}\right)^{2}(K / 2)^{2}=\left(\frac{\pi}{n}\left\lfloor\frac{n \epsilon}{\pi}\right\rfloor\right)^{2} .
\end{aligned}
$$

That is, we obtain

$$
C_{\theta_{0}}\left(\frac{1}{\sqrt{2}}(|n, 0\rangle+|0, n\rangle): \epsilon\right) \geq\left(\frac{\pi}{n}\left\lfloor\frac{n \epsilon}{\pi}\right\rfloor\right)^{2} .
$$

The lower bound $\left(\frac{\pi}{n}\left\lfloor\frac{n \epsilon}{\pi}\right\rfloor\right)^{2}$ converges $\epsilon^{2}$. This means that the mini-max of the mean square error does not decrease when $n$ increase. Hence,

$$
\lim _{n \rightarrow \infty} n^{2} C_{\theta_{0}}\left(\frac{1}{\sqrt{2}}(|n, 0\rangle+|0, n\rangle): \epsilon\right)=\infty .
$$

Next, we consider the asymptotic limit of the performance with the global asymptotic min-max criterion when the noon state $\frac{1}{\sqrt{2}}(|n, 0\rangle+|0, n\rangle)$. By using (20) and (7), the relation

$$
\lim _{n \rightarrow \infty} n^{2} C\left(\frac{1}{\sqrt{2}}(|n, 0\rangle+|0, n\rangle)\right)=\infty
$$

holds. This fact can be shown in another method based on (13). In fact, the $L^{2}$ function corresponding to the noon state via $(10)$ is $f_{0}(x):=\frac{1}{\sqrt{2}}(\delta(x-1)+\delta(x+1))$. The function $f_{\eta}(x):=\frac{1}{\sqrt{2}}\left(\delta(x-1)+e^{i \eta} \delta(x+1)\right)$ satisfies $\left\langle f_{\eta}\left|P^{2}\right| f_{\eta}\right\rangle=\infty$. Thus, the relation (13) yields (21).

Next, we replace the number state by the coherent state. In the following, the coherent state with complex amplitude $\alpha_{1}, \alpha_{2}$ is written as $\left|\alpha_{1}, \alpha_{2}\right\rangle_{c}$. Then, we 
consider the case when the covariant measurement is applied and we input the noon state $\frac{1}{\sqrt{2}}\left(|n, 0\rangle_{c}+|0, n\rangle_{c}\right)$, which is called the coherent noon state. The $L^{2}$ function corresponding to the coherent noon state via (10) is the same function $f_{0}$. Hence, the relation (13) yields

$$
\lim _{n \rightarrow \infty} n^{2} C\left(\frac{1}{\sqrt{2}}\left(|n, 0\rangle_{c}+|0, n\rangle_{c}\right)\right)=\infty .
$$

\section{Conclusion}

We have discussed three kinds of bounds of MSE in the phase estimation with photon number constraints. The first is the global minimax bound. The second is the local minimax bound. The third is the bound with the locally unbiased condition. These bounds have been treated with two kinds of constraints: One is the constraint for the average of the square of the photon number concerning the input state. The other is the constraint for the maximum photon number concerning the input state. We have shown that the asymptotic limits $C_{a v}, C_{a v, \theta}$, and $\tilde{C}_{a v, \theta}$ of three kinds of bounds coincide under the first constraint. However, these bounds $C_{\text {max }}, C_{\text {max }, \theta}$, and $\tilde{C}_{\text {max }, \theta}$ do not coincide under the second kind of constraint. In fact, the locally unbiased condition and Fisher information are originally mathematical concepts. In the independent and identical distributed setting of state estimation, these values provide the asymptotic bound of MSE [12]-[14]. However, these values do not provide the operational meaning in general. That is, these values have no operational meaning when they do not coincide with operational values. Hence, under the second kind of constraint, only these bounds $C_{\max }$ and $C_{\max , \theta}$ have the real meaning.

Further, we should be careful of the meaning of the noon state and the coherent noon state. When these states are inputed, the optimal SLD Fisher information is realized. However, under the local mini-max criterion, as is discussed in section 6, the MSE of the noon input state does not convergence to zero, and the noon input state is far from the optimal input. The optimal input is given in (14) and (15). Hence, in order to realize the high performance phase estimation, it is desired to implement the input states (14) and (15).

\section{Acknowledgement}

The author was partially supported by a Grant-in-Aid for Scientific Research in the Priority Area 'Deepening and Expansion of Statistical Mechanical Informatics (DEX-SMI)', No. 18079014 and a MEXT Grant-inAid for Young Scientists (A) No. 20686026. The Centre for Quantum Technologies is funded by the Singapore Ministry of Education and the National Research Foundation as part of the Research Centres of Excel- lence programme. The author thanks Dr. William J. Munro and Mr. Akihito Soeda for helpful comments. He also thanks the referees for helpful comments concerning this manuscript.

\section{References}

[1] A. Luis and J. Perina, "Optimum phase-shift estimation and the quantum description of the phase difference," Phys. Rev. A, vol.54, p.4564, 1996.

[2] V. Buzek, R. Derka, and S. Massar, "Optimal quantum clocks,” Phys. Rev. Lett., vol.82, p.2207, 1999.

[3] H. Imai and M. Hayashi, "Fourier Analytic Approach to Phase Estimation in Quantum Systems," New J. Phys., vol.11, 043034, 2009.

[4] A. Y. Kitaev, A. H. Shen, and M. N. Vyalyi, Classical and Quantum Computation, (Graduate Studies in Mathematics 47), American Mathematical Society, 2002.

[5] V. Giovannetti, S. Lloyd, and L. Maccone, "Quantumenhanced measurements: beating the standard quantum limit," Science, vol.306, pp.1330-1336, 2004.

[6] V. Giovannetti, S. Lloyd, and L. Maccone, "Quantumenhanced "Quantum metrology"," Phys. Rev. Lett., vol.96, 010401, 2006.

[7] B. L. Higgins, D. W. Berry, S. D. Bartlett, H. M. Wiseman, and G. J. Pryde, "Entanglement-free Heisenberg-limited phase estimation," Nature, vol.450, pp.393-396, 2007.

[8] T. Nagata, R. Okamoto, J. L. O'Brien, K. Sasaki, and S. Takeuchi, "Beating the Standard Quantum Limit with Four-Entangled Photons," Science, vol.316, no.5825, p.726, 2007.

[9] R. Okamoto, H. F. Hofmann, T. Nagata, J. L. O’Brien, K. Sasaki, and S. Takeuchi, "Beating the standard quantum limit: phase super-sensitivity of N-photon interferometers," New J. Phys., vol.10, 073033, 2008.

[10] J. A. Jones, S. D. Karlen, J. Fitzsimons, A. Ardavan, S. C. Benjamin, G. A. D. Briggs, and J. J. L. Morton, "Magnetic Field Sensing Beyond the Standard Quantum Limit Using 10-Spin NOON States," Science, vol.324, pp.1166-1168, 2009.

[11] L. Pezze, A. Smerzi, G. Khoury, J. F. Hodelin, and D. Bouwmeester, "Phase Detection at the Quantum Limit with Multiphoton Mach-Zehnder Interferometry," Phys. Rev. Lett., vol.99, 223602, 2007.

[12] M. Hayashi and K. Matsumoto, "Statistical model with measurement degree of freedom and quantum physics," RIMS koukyuroku, no 1055, Kyoto, Kyoto University, p.96, 1998, In Japanese; M. Hayashi and K. Matsumoto, Asymptotic Theory of Quantum Statistical Inference, Ed M. Hayashi, Singapore, World Scientific, p.162, 2005, reprinted, English translation.

[13] R. Gill and S. Massar, "State estimation for large ensembles," Phys. Rev. A, vol.61, 042312, 2000. 
[14] A. Fujiwara, "Strong consistency and asymptotic efficiency for adaptive quantum estimation problems," $J$. Phys. A: Math. Gen., vol.39, p.12489, 2006.

[15] C. W. Helstrom, Quantum Detection and Estimation Theory, New York, Academic Press, 1976.

[16] A. S. Holevo, Probabilistic and Statistical Aspects of Quantum Theory, Amsterdam, North-Holland, 1982, (Originally published in Russian 1980).

[17] M. Hayashi, Quantum Information: An Introduction, Berlin, Springer, 2006.

[18] A. S. Holevo, "Covariant measurements and uncertainty relations," Rep. Math. Phys., vol.16, pp.385-400, 1979.

[19] J. Hajek, "Local asymptotic minimax and admissibility in estimation," Proc. Sixth Berkeley Symp. on Math. Statist. and Prob., vol.1, Univ. of Calif. Press, pp.175194, 1972.

[20] M. Hayashi, "Comparison between the Cramer-Rao and the mini-max approaches in quantum channel estimation," to be published in Communications in Mathematical Physics, arXiv:1003.4575.

[21] A. Fujiwara and H. Nagaoka, "Quantum Fisher metric and estimation for pure state models," Phys. Lett. A, vol.201, p.119, 1995.

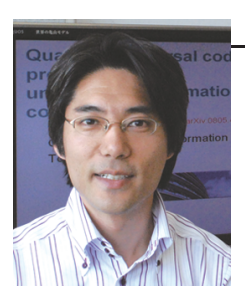

Masahito HAYASHI

Masahito HAYASHI was born in Japan in 1971. He received the B.S. degree from the Faculty of Sciences in Kyoto University, Japan, in 1994 and the M.S. and Ph.D. degrees in Mathematics from Kyoto University, Japan, in 1996 and 1999, respectively. He worked in Kyoto University as a Research Fellow of the Japan Society of the Promotion of Science (JSPS) from 1998 to 2000, and worked in the Laboratory for Mathematical Neuroscience, Brain Science Institute, RIKEN from 2000 to 2003, and worked in ERATO Quantum Computation and Information Project, Japan Science and Technology Agency (JST) as the Research Head from 2003 to 2007. He also worked in the Superrobust Computation Project Information Science and Technology Strategic Core (21st Century COE by MEXT) Graduate School of Information Science and Technology, The University of Tokyo as an Adjunct Associate Professor from 2004 to 2007. In 2006, he published the book "Quantum Information: An Introduction" from Springer. In 2007, he joined the Graduate School of Information Sciences, Tohoku University as an Associate Professor. He also worked at Centre for Quantum Technologies, National University of Singapore, as a Visiting Research Associate Professor from 2009. His research interests include quantum information theory and quantum statistical inference. Dr. Hayashi is on the Editorial Board of International Journal of Quantum Information and International Journal On Advances in Security. In 2010, He was awarded the Japan IBM prize with computer science section. 